\title{
Short communication: Automated testing of accelerometer transverse sensitivity with a flexure-based XY compliant stage
}

\author{
Tiemin $\mathrm{Li}^{1}{ }^{1,}$, Yunsong $\mathrm{Du}^{1, *}$, Wei $\mathrm{Ji}^{2}$, Zhihua $\mathrm{Liu}^{3}$, and Chenguang $\mathrm{Cai}^{3}$ \\ ${ }^{1}$ Manufacturing Engineering Institute, Department of Mechanical Engineering, Tsinghua University, Beijing, \\ 100084, China \\ ${ }^{2}$ Department of Foreign Language Hebei Normal University for Nationality, Chengde, 067000, China \\ ${ }^{3}$ Division of Mechanics and Acoustics, National Institute of Metrology, Beijing, 100029, China \\ *These authors contributed equally to this work.
}

Correspondence to: Yunsong Du (duyunsongwei@163.com)

Received: 22 March 2016 - Revised: 18 July 2016 - Accepted: 18 July 2016 - Published: 2 August 2016

\begin{abstract}
This paper presents the automated testing of accelerometer transverse sensitivity with a flexure-based compliant stage which can provide vibration on the XY plane. An experimental platform is set up, and the circular tracking error is first obtained to verify the feasibility of the stage. The transverse sensitivity of a threeaxis piezoelectric accelerometer is subsequently tested, and it is below $3 \%$. The difference of the transverse sensitivity under various frequencies is $0.4 \%$, while the difference of measured direction angle is 2.49 , which validates the accuracy of the proposed method.
\end{abstract}

\section{Introduction}

Piezoelectric accelerometers possess many advantages, such as compact size, small mass, simple structure, and fast response time (Jiang et al., 2013). Owing to these advantages, they are widely used as the vibration sensor. A number of calibration tests including the transverse sensitivity should be performed by the manufacturer to ensure the quality and accuracy of the accelerometers (Wang et al., 2012; Dosch and Lally, 2015). Calibration methods for testing of accelerometer transverse sensitivity had been listed in the international standard ISO 16063-31. It included the standards using a single-axis vibration generator, a vibration generator with turntable, a two-axis vibration generator, and a three-axis vibration generator (ISO 16063-31:2009).

Compared with the single-axis vibration generator, the two-axis vibration generator can provide more accurate, automatic and consistent calibration (Dosch and Lally, 2015). However, in practice, only the resonant beam and double turntable centrifuge are developed as two-axis vibration generators (Dosch and Lally, 2015; Guan et al., 2015; Umeda et al., 2004). Their synchronous control and test accuracy are still challenges (Umeda et al., 2004; Lee and Kim, 2012), and the range of application is limited (Guan et al., 2015). Therefore, it is necessary to propose a new two-axis method for testing accelerometer transverse sensitivity.

This paper addresses a two-axis method for testing of accelerometer transverse sensitivity with a flexure-based compliant stage, which can be a supplement to the existing twoaxis methods. It is the first time to apply the compliant stage to test accelerometer transverse sensitivity. The remaining sections are organized as follows. In Sect. 2, the detailed structure of the stage is presented. In Sect. 3, the principle of testing transverse sensitivity with the proposed stage is introduced. In Sect. 4, experiments have been done, including the circular trajectory tests and the testing of accelerometer transverse sensitivity. Finally, conclusions are drawn in Sect. 5.

\section{The flexure-based compliant stage}

Recently, numbers of compliant stages have been developed (Sun et al., 2013; Hao and Yu, 2016; Yu et al., 2015; Hao and 
Kong, 2012). To generate enough vibration, a 2-degree-offreedom flexure-based stage is designed. The constant rectangular cross-section flexure hinges are selected, and the detailed structure of the flexure-based stage is illustrated in Fig. 1. The motivation of the design is to obtain high rigidity and high compactness. The proposed stage is designed with four kinematic limbs. In each kinematic limb, two serially connected prismatic joints $P_{1}$ and $P_{2}$ are designed. The prismatic joint $P_{2}$ is connected to the fixed base directly, and it has the input platform which can provide the forces actuated by the piezoelectric actuators (PZTs) to the micro-motion stage and connect to the prismatic joint $P_{1}$. The prismatic joint $P_{1}$ connected to the output platform could transmit the deformations of the flexure hinges to the output platform.

The proposed flexure-based compliant stage should possess high resonance frequency, large stiffness, good decoupled property, and enough workspace. In addition, PZTs are selected to drive the stage because they can provide fast and ultra-precision motions. The design objective is to have high resonance frequency. In practice, the flexure-based compliant stage is composed of various flexure hinges, and their performances are easily influenced by their geometrical parameters. The parameters are thus necessary to be optimized. Meantime, to generate enough vibration, the minimum workspace is $18 \mu \mathrm{m}$. In our earlier paper (Du et al., 2016), the design and optimization have been discussed. Compared with the design presented in Sun et al. (2013), Hao and Yu (2016), Yu et al. (2015), and Hao and Kong (2012), the prismatic joints are designed according to the divided parts $n$ of the flexure hinge thickness. In addition, the distance between two neighboring hinges of the prismatic joints is considered in the dimensional optimization. Meanwhile, it can get relative high resonance frequency. The optimized parameters are listed as follows: $L_{1}=25 \mathrm{~mm}, L_{2}=$ $28 \mathrm{~mm}, t_{1}=2.5 \mathrm{~mm}, t_{2}=3 \mathrm{~mm}, d_{1}=11.5 \mathrm{~mm}$ and $d_{2}=$ $11 \mathrm{~mm}$. The thickness of the stage is chosen as $10 \mathrm{~mm}$, and the output platform and input platform are both selected as $20 \mathrm{~mm} \times 20 \mathrm{~mm}$.

\section{Principle of testing transverse sensitivity}

The transverse sensitivity of an accelerometer is the sensitivity to acceleration applied at right angles (on the perpendicular plane) to its geometric axis (Dosch and Lally, 2015; ISO 16063-31:2009; Guan et al., 2015). In fact, the sensitive axis of the accelerometer is not necessarily aligned with the geometric axis. The principle of testing transverse sensitivity with the proposed stage can be demonstrated in Fig. 2 . The sinusoidal signals are applied to the PZTs. The flexurebased compliant stage can be actuated by the PZTs to obtain relative high-frequency motion on the XY plane.

The motion is controlled such that the displacement along the $x$ and $y$ axis are shifted in phase by $90^{\circ}$. The initial phase of the sinusoidal signals is selected as zero, and the output

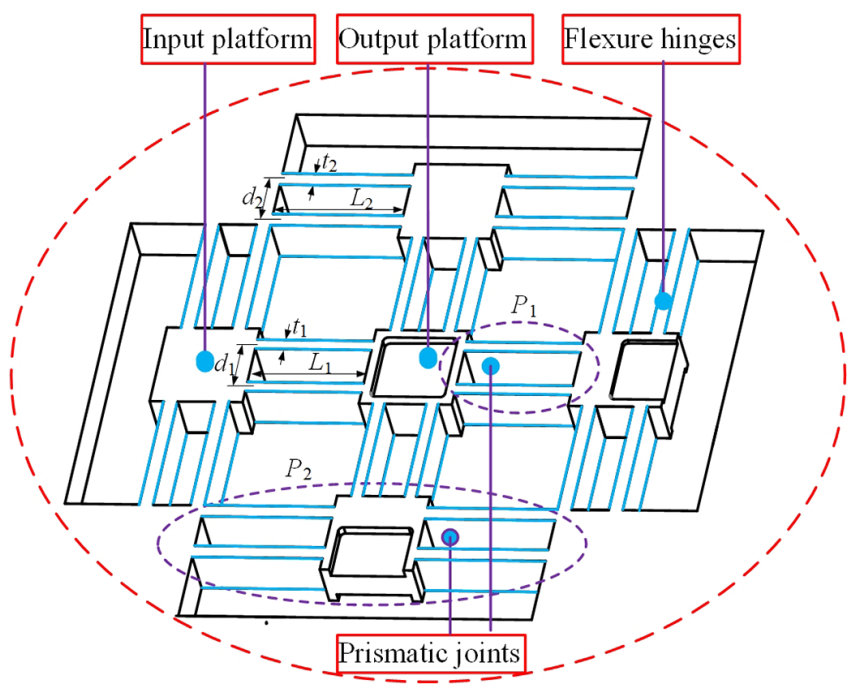

Figure 1. The detailed structure of the flexure-based stage.

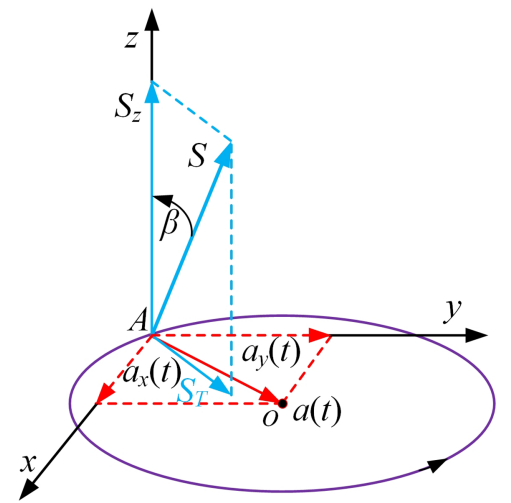

Figure 2. The principle of testing transverse sensitivity.

displacement of the compliant stage can be written as

$L=\left[\begin{array}{l}L_{x}(t) \\ L_{y}(t) \\ L_{z}(t)\end{array}\right]=\left[\begin{array}{l}M_{x} \cos \left(2 \pi f_{x} t\right) \\ M_{y} \sin \left(2 \pi f_{y} t\right) \\ 0\end{array}\right]$,

where $M_{x}$ and $M_{y}$ are the amplitude of the sinusoidal signals, $f_{x}$ and $f_{y}$ are the frequency of the sinusoidal signals, and $t$ is the time.

The acceleration components along the $x$ and $y$ axis could be generated in conjunction with consideration of the reciprocating motion. The acceleration components $a_{x}(t)$ and $a_{y}(t)$ could be combined to an elliptical motion on the $\mathrm{XY}$ plane. Without loss of generality, a circular motion could also be obtained when the acceleration components $a_{x}(t)$ and $a_{y}(t)$ have equal frequency $f\left(f=f_{x}=f_{y}\right)$ and amplitude $M\left(M=M_{x}=M_{y}\right)$. Then, the output acceleration 


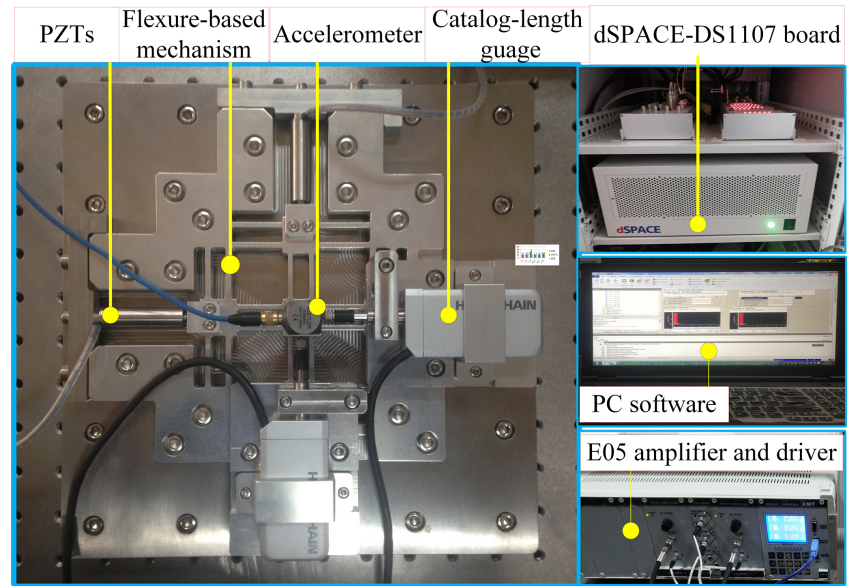

Figure 3. The experimental platform of the flexure-based compliant stage.

of the flexure-based compliant stage can be expressed as

$a=\left[\begin{array}{l}a_{x}(t) \\ a_{y}(t) \\ a_{z}(t)\end{array}\right]=\left[\begin{array}{l}-4 \pi^{2} f^{2} M \cos (2 \pi f) \\ -4 \pi^{2} f^{2} M \sin (2 \pi f) \\ 0\end{array}\right]$.

Thus, the acceleration components $a_{x}(t)$ and $a_{y}(t)$ can be combined to the resultant centripetal acceleration. The magnitude and the direction angle determined at any time $t$ can be expressed as

$a(t)=\sqrt{\left(a_{x}(t)\right)^{2}+\left(a_{y}(t)\right)^{2}}=4 \pi^{2} f^{2} M$

$\theta(t)=\tan ^{-1}\left(a_{y}(t) / a_{x}(t)\right)$.

According to the standard ISO 16063-31, the transverse sensitivity is expressed as a percentage of the geometric axis sensitivity, and the transverse sensitivity of the accelerometer under test can be expressed as

$S_{t}=\max (u(t) / a(t))$,

where $u(t)$ is the output of the accelerometer under test.

The transverse sensitivity should be minimized. It is usually below $5 \%$ for a large number of piezoelectric accelerometers, while it is below 3\% for those with higher precision (Dosch and Lally, 2015). The stage based two-axis vibration generator has many advantages such as high precision, high efficiency, low cost and ease of control. Furthermore, it can directly obtain the transverse sensitivity and the corresponding angle simultaneously.

\section{Experimentation and results}

The apparatus of the flexure-based compliant stage are illustrated in Fig. 3. The stage is fabricated by the milling process with aluminum 7075, and it is actuated by two preselected PZTs (PSt 150/7/40 VS12 from XMT Harbin, Inc.).

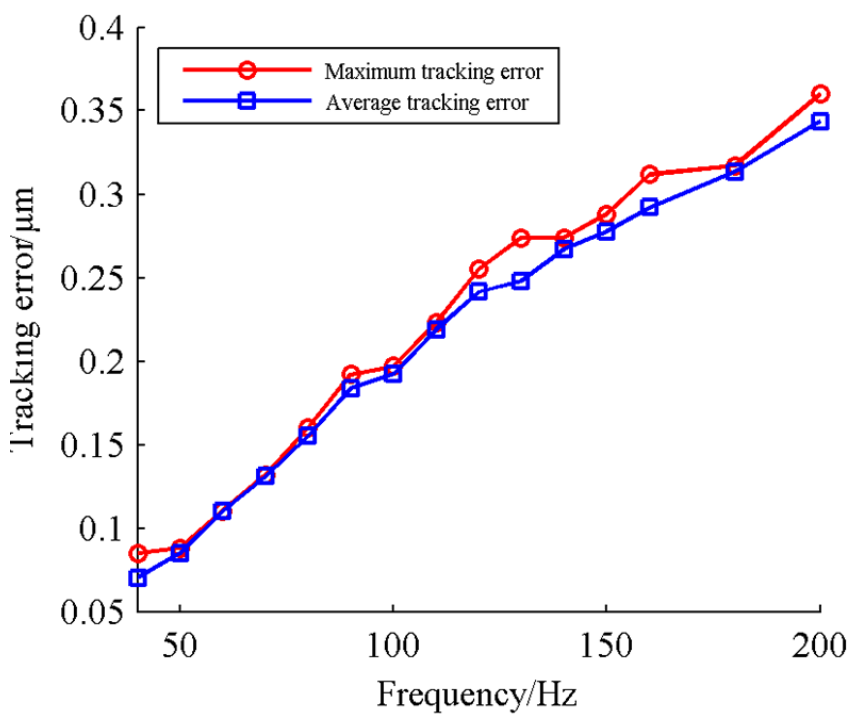

Figure 4. Tracking error for circular trajectories under various input frequencies.

The two-axis piezo amplifier and driver (XE500/E05 from XMT Harbin, Inc.) is used to provide excitation voltage (0$120 \mathrm{~V}$ ). The catalog-lengthe gauges (MT 1281 from Heidenhain, Inc.) are adopted to measure the displacements. In addition, a dSPACE-DS1107 board and the corresponding PC software are used to control the whole system. The first frequency of the flexure-based compliant stage is $1987 \mathrm{~Hz}$, and it can be actuated by the PZTs at relative high frequency between 0 to $200 \mathrm{~Hz}$. The largest input amplitude of the sinusoidal signals is selected as $5 \mu \mathrm{m}$ to get high-precision tracking performance. Therefore, the acceleration generated by the flexure-based compliant stage should be ranged from 0 to $7.89 \mathrm{~m} \mathrm{~s}^{-2}$.

A proportional-integral (PI) controller could be implemented on the controller to close the position loop. The dSPACE-DS1107 board is equipped with the 16-bit analog to digital converters and 16-bit digital to analog converters to control the whole system. The proposed board can receive displacement feedback from the catalog-length gauges, and then send it to the E05 amplifier and driver. The amplified voltage can be provided by the amplifier to drive the PZTs. The synchronous control and motion is obtained because the symmetrical configuration of the flexure-based stage can possess same performances along $x$ and $y$ axis.

\subsection{Circular trajectory tests}

To verify the feasibility of the stage, the circular tracking error under various input frequencies is studied, as demonstrated in Fig. 4. In practice, the dynamic performances of the compliant stage could be influenced by the load mass on the output platform. Therefore, the circular tracking error of the compliant stage installing the accelerometer un- 

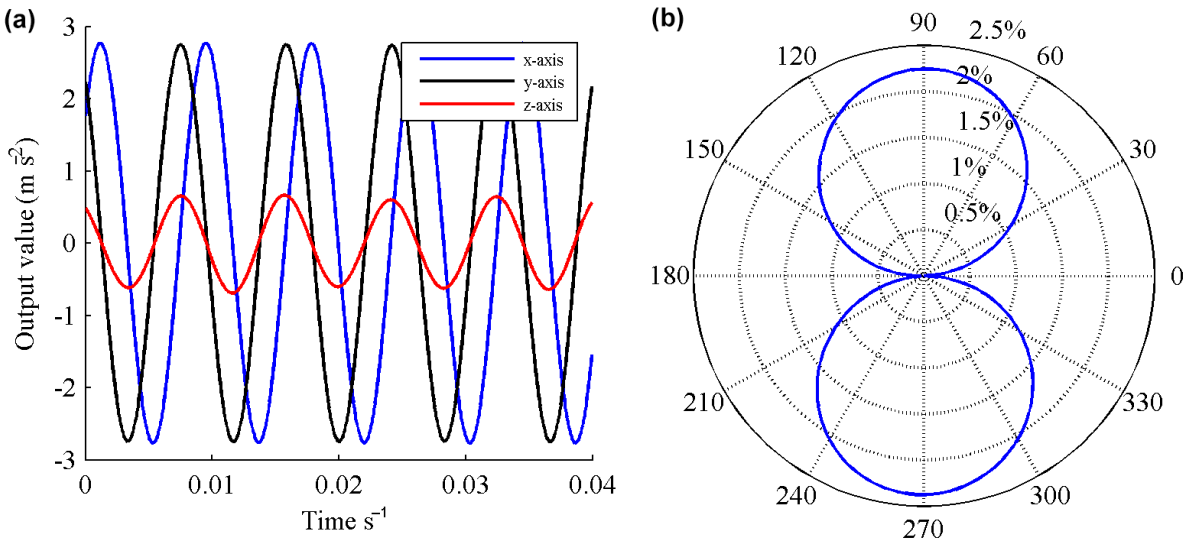

Figure 5. Experimental results under test at $120 \mathrm{~Hz}$ : (a) show the output; (b) show the polar figures of the relative transverse sensitivity.

Table 1. Results of testing transverse sensitivity under various input frequencies.

\begin{tabular}{cccc}
\hline Input frequency $(\mathrm{Hz})$ & Vibration amplitude $\left(\mathrm{m} \mathrm{s}^{-2}\right)$ & Transverse sensitivity & Direction angle $\left(^{\circ}\right)$ \\
\hline 80 & 1.263 & $2.61 \%$ & 90.56 \\
90 & 1.598 & $2.62 \%$ & 88.07 \\
110 & 2.388 & $2.35 \%$ & 89.23 \\
120 & 2.842 & $2.37 \%$ & 88.30 \\
130 & 3.336 & $2.22 \%$ & 88.63 \\
140 & 3.868 & $2.23 \%$ & 90.50 \\
\hline
\end{tabular}

der test should be analyzed first. The load mass of the accelerometer under test is $15.276 \mathrm{~g}$, and the circular radius is selected as $5 \mu \mathrm{m}$. We can see that the maximum tracking error is larger than the average tracking error with increasing frequency from 40 to $200 \mathrm{~Hz}$. Comparing with the maximum tracking error which can be changed by altering the PI parameters, the average tracking error can be used to represent the actual tracking error. It shows that the average tracking error exhibits an upward trend from $0.07 \mu \mathrm{m}(1.4 \%)$ to $0.34 \mu \mathrm{m}(6.8 \%)$. According to the international standard ISO 16063-31, the circular tracking error of the vibration generator should be below $5 \%$, and thus the transverse sensitivity of the selected accelerometer should be measured under $140 \mathrm{~Hz}$.

\subsection{Accelerometer transverse sensitivity tests}

A three-axis piezoelectric accelerometer (KISTLER 8766A50) is employed to test the transverse sensitivity along the $z$ axis. The $z$ axis is perpendicular to the XY plane. The proposed accelerometer under test can be used to receive the vibration signals along $x, y$, and $z$ axis. The data acquisition and analysis are performed using a modal analyzer (Coinv INV 3018C) and the corresponding DASP software.

As shown in Fig. 5, the output of the accelerometer under test and the polar figure of the corresponding transverse sensitivity with the input frequency of $120 \mathrm{~Hz}$ are illustrated. The output value along $x$ and $y$ axis is on the XY plane, and the output along $z$ axis is perpendicular to the XY plane. As the output signal along $z$ axis is much less than that on the $X Y$ plane, the output signal along $z$ axis is amplified 10 times for better illustration. Obviously, the polar figure of transverse output is like the typical " 8 " figure depicted in ISO standard 16063-31. And the " 8 " figure is almost symmetrical. It can be seen that the vibration amplitude is $2.84 \mathrm{~m} \mathrm{~s}^{-2}$, and the transverse sensitivity is $2.37 \%$.

The results of transverse sensitivity under various input frequencies are illustrated in Table 1 . The maximum difference of the measured transverse sensitivity is $0.4 \%$. In theory, the polar figure of the transverse sensitivity is symmetrical. Therefore, the angle results should be subtracted 180 to get the acute angles. The maximum difference of the measured direction angle is 2.49. Similarly, the transverse sensitivity of the selected three-axis accelerometer along the $x$ and $y$ axis can be measured using the proposed method.

\section{Conclusions}

This paper presented a method for testing the accelerometer transverse sensitivity at relative high frequency. The principle of the method was analyzed. To verify the feasibility of the proposed method, circular trajectories tests were carried out. A three-axis piezoelectric accelerometer was selected to test its transverse sensitivity. Based on the results obtained, we can draw the following conclusions: 
The circular tracking error is below 5\% (from 0 to $140 \mathrm{~Hz}$ ), which verifies the feasibility of the proposed method.

It shows that the transverse sensitivity of the selected piezoelectric accelerometer is below $3 \%$, the maximum difference of the transverse sensitivity is $0.4 \%$, and the maximum difference of the measured direction angle is 2.49 , which validates the accuracy of the proposed method.

The flexure-based XY compliant stage, which can provide enough vibration on the XY plane, could be applied to test accelerometer transverse sensitivity. And it is a supplement to the current standard methods.

Acknowledgements. This work was supported by the National Science and Technology Major Project of China (grant no. 2015ZX04014021 and grant no. 2015ZX04001002), the National Science Foundation of China (grant no. 51275260), and the Tsinghua University Initiative Scientific Research Program (no. 2014z22068).

Edited by: Guangbo Hao

Reviewed by: P. Yan and one anonymous referee

\section{References}

Dosch, J. J. and Lally, D. M.: Automated testing of accelerometer transverse sensitivity, available as article reprint AR-69 from PCB Piezotronics, Inc., http://www.pcb.com, last access: 5 October 2015.

Du, Y. S., Li, T. M., Jiang, Y., and Wang, H. T.: Design and analysis of a 2-degree-of-freedom flexure-based micro-motion stage, Adv. Mech. Eng., 8, 1-13, doi:10.1177/1687814016638301, 2016.

Guan, W., Meng, X. F., and Dong, X. M.: Testing Transverse Sensitivity of Linear Single-Axis Pendulous Accelerometer with Double Turntable Centrifuge, Mapan, J. Metrol. Soc., 31, 1-6, doi:10.1007/s12647-015-0151-3, 2015.
Hao, G. and Kong, X.: A novel large-range XY compliant parallel manipulator with enhanced out-of-plane stiffness, J. Mech. Design, 6, 061009, doi:10.1115/1.4006653, 2012.

Hao, G. and Yu, J.: Design, modelling and analysis of a completely-decoupled XY compliant parallel manipulator, Mech. Mach. Theory, 102, 179-195, doi:10.1016/j.mechmachtheory.2016.04.006, 2016.

ISO 16063-31: Methods for the calibration of vibration and shock transducers-Part 31: Testing of transverse vibration sensitivity, 2009.

Jiang, X., Kim, K., Zhang, S., Johnson, J., and Salazar, G.: High-temperature piezoelectric sensing, Sensors, 14, 144-169, doi:10.3390/s140100144, 2013.

Lee, D. H. and Kim, B. Y.: Multiple-reflection interferometer for high accuracy measurement of small vibration displacement, Rev. Sci. Instrum, 71, 1981-1986, doi:10.1063/1.1150565, 2000

Sun, X., Chen, W. H., Zhou, R., Chen, W. J., and Zhang, J. B.: A decoupled 2-DOF flexure-based micropositioning stage with large travel ranges, Robotica, 32, 677-694, doi:10.1017/S0263574713000969, 2013.

Umeda, A., Onoe, M., Sakata, K., Fukushia, T., Kanari, K., Lioka, H., and Kobayashi, T.: Calibration of three-axis accelerometers using a three-dimensional vibration generator and three laser interferometers, Sens. Actuator A-Phys., 114, 93-101, doi:10.1016/j.sna.2004.03.011, 2004.

Wang, Q., Zhang, D. W., Yang, H. Y., Tao, C. X., Huang, Y. S. Zhuang, S. L., and Mei, T.: Sensitivity of a label-free guidedmode resonant optical biosensor with different modes, Sensors, 12, 9791-9799, doi:10.3390/s120709791, 2012.

Yu, J., Xie, Y., Li, Z, and Hao, G.: Design and experimental testing of an improved large-range decoupled XY compliant parallel micromanipulator, J. Mech. Robot, 4, 044503, doi:10.1115/1.4030467, 2015. 